\title{
Crux of Using the Cascaded Emission of a Three-Level Quantum Ladder System to Generate Indistinguishable Photons
}

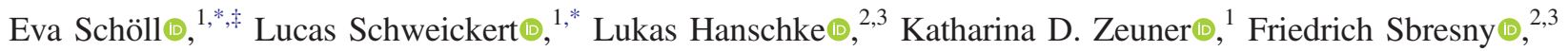 \\ Thomas Lettner $\odot,{ }^{1}$ Rahul Trivedi, ${ }^{4}$ Marcus Reindl®,${ }^{5}$ Saimon Filipe Covre da Silva, ${ }^{5}$ Rinaldo Trotta $\odot,{ }^{6}$ Jonathan \\ J. Finley, ${ }^{7,3}$ Jelena Vučković $\odot,{ }^{4}$ Kai Müller@ ${ }^{2,3}$ Armando Rastelliø, ${ }^{5}$ Val Zwiller $\odot,{ }^{1}$ and Klaus D. Jöns $\oplus^{1, \dagger, \$}$ \\ ${ }^{1}$ Department of Applied Physics, Royal Institute of Technology, Albanova University Centre, \\ Roslagstullsbacken 21, 10691 Stockholm, Sweden \\ ${ }^{2}$ Walter Schottky Institut and Department of Electrical and Computer Engineering, Technische Universität München, \\ 85748 Garching, Germany \\ ${ }^{3}$ Munich Center for Quantum Science and Technology, Schellingstr. 4, 80799 Munich, Germany \\ ${ }^{4}$ Ginzton Laboratory, Stanford University, Stanford, California 94305, USA \\ ${ }^{5}$ Institute of Semiconductor and Solid State Physics, Johannes Kepler University Linz, 4040 Linz, Austria \\ ${ }^{6}$ Dipartimento di Fisica, Sapienza Università di Roma, Piazzale A. Moro 1, I-00185 Roma, Italy \\ ${ }^{7}$ Walter Schottky Institut and Physik Department, Technische Universität München, 85748 Garching, Germany
}

(Received 11 June 2020; accepted 22 October 2020; published 1 December 2020)

\begin{abstract}
We investigate the degree of indistinguishability of cascaded photons emitted from a three-level quantum ladder system; in our case the biexciton-exciton cascade of semiconductor quantum dots. For the threelevel quantum ladder system we theoretically demonstrate that the indistinguishability is inherently limited for both emitted photons and determined by the ratio of the lifetimes of the excited and intermediate states. We experimentally confirm this finding by comparing the quantum interference visibility of noncascaded emission and cascaded emission from the same semiconductor quantum dot. Quantum optical simulations produce very good agreement with the measurements and allow us to explore a large parameter space. Based on our model, we propose photonic structures to optimize the lifetime ratio and overcome the limited indistinguishability of cascaded photon emission from a three-level quantum ladder system.
\end{abstract}

DOI: 10.1103/PhysRevLett.125.233605

Indistinguishable photons are one of the most essential resources in photonic quantum technologies since they mediate photon-photon interactions by the Hong-OuMandel effect [1] needed for quantum information processing [2], quantum sensing [3], and quantum networks [4]. Although probabilistic generation of indistinguishable photon pairs by parametric down-conversion has been used as the work horse in quantum optics experiments and in proof-of-principle applications, there is a strong need for the on-demand generation of near-unity indistinguishable photons. This need has led to a whole research field investigating novel solid-state quantum emitters [5], optimizing all relevant parameters to fabricate the ideal quantum light source. One promising quantum light source to reach these goals are epitaxially grown semiconductor quantum dots (QDs), emitting on-demand [6] near unity indistinguishable single photons $[7,8]$. QDs have recently

Published by the American Physical Society under the terms of the Creative Commons Attribution 4.0 International license. Further distribution of this work must maintain attribution to the author(s) and the published article's title, journal citation, and DOI. Funded by Bibsam. been used to perform photonic quantum simulations [9-11] as well as photonic quantum sensing [12,13]. In addition, QDs are the only quantum emitter able to generate ondemand polarization entangled photon pairs [14], using the biexciton-exciton cascade [15]. This puts QDs on the map as ideal quantum light sources to realize quantum relays [16-18] and quantum repeaters based on the Shapiro Lloyd scheme [19]. However, these applications require the simultaneous generation of near-unity indistinguishable and maximally entangled photon pairs. Despite, large research efforts [14,20-22] achieving highly indistinguishable photons from the biexciton-exciton cascade has proven to be elusive even under optimized excitation conditions [23]. Here, we show that this stems from the intrinsic properties of the quantum three-level ladder system, inherent to QDs, which reduces the maximally achievable indistinguishability of the emitted photons. To this end, we first demonstrate analytically that the indistinguishability is identical for the emission from either of the cascaded transitions, albeit limited. This finding is then tested experimentally by measuring the photon indistinguishability for emission from a quantum two-level system and a quantum three-level ladder system using the same quantum emitter. Here, we evaluate data from four different 
QDs and extract the relevant parameters. Finally, we perform quantum-optical simulations exploring a wide parameter space. The results are in good agreement with our measurements. To overcome the limitations of threelevel quantum ladder systems we propose nanoengineering the lifetimes of the involved excited states.

To gain theoretical insight into the fundamental limits of the trace purity of photons obtained from the cascaded twophoton emission, we consider a three-level quantum ladder system with ground state $|G\rangle$, intermediate state $|X\rangle$, and excited state $|\mathrm{XX}\rangle$. Using a short laser pulse, the system is initialized to the excited state $|\mathrm{XX}\rangle$ at $t=0$ and allowed to decay from $|\mathrm{XX}\rangle \rightarrow|X\rangle \rightarrow|G\rangle$ via the cascaded emission of two photons. The emitted two-photon state can be easily computed using a scattering matrix formalism $[24,25]$ as

$|\psi\rangle=\int_{t=0}^{\infty} \int_{t^{\prime}=t}^{\infty} d t d t^{\prime} f\left(t, t^{\prime}\right) b_{\mathrm{XX} \rightarrow X}^{\dagger}(t) b_{X \rightarrow G}^{\dagger}\left(t^{\prime}\right)|\mathrm{vac} ; G\rangle$,

where $b_{\mathrm{XX} \rightarrow X}^{\dagger}(t)$ and $b_{X \rightarrow G}^{\dagger}(t)$ are the time-domain creation operators describing the photonic modes that the transitions $|\mathrm{XX}\rangle \rightarrow|X\rangle$ and $|X\rangle \rightarrow|G\rangle$ couple to, and $f\left(t, t^{\prime}\right)$ is the two-photon wave function of the emitted photon given by

$f\left(t, t^{\prime}\right)=\sqrt{\gamma_{X} \gamma_{\mathrm{XX}}} e^{-\mathrm{i} \omega_{\mathrm{XX} \rightarrow X} t} e^{-\mathrm{i} \omega_{X \rightarrow G} t^{\prime}} e^{-\gamma_{X}\left(t^{\prime}-t\right) / 2} e^{-\gamma_{\mathrm{XX}} t / 2}$,

where $\omega_{\mathrm{XX} \rightarrow X}, \omega_{X \rightarrow G}$ are the frequencies of the transitions $|\mathrm{XX}\rangle \rightarrow|X\rangle,|X\rangle \rightarrow|G\rangle$ and $\gamma_{\mathrm{XX}}, \gamma_{X}$ are the decay rates of the states $|\mathrm{XX}\rangle,|X\rangle$. The single photon emitted from the transition $|\mathrm{XX}\rangle \rightarrow|X\rangle$ can then be described by a mixed state obtained by tracing the pure two-photon state $(|\psi\rangle\langle\psi|)$ over the modes described by operator $b_{X \rightarrow G}(t)$. The density matrix of this state is calculated from Eq. (1a):

$$
\begin{aligned}
\rho & =\operatorname{Tr}_{b_{X \rightarrow G}}[|\psi\rangle\langle\psi|] \\
& =\int_{0}^{\infty} \int_{0}^{\infty} d t d t^{\prime} \rho\left(t, t^{\prime}\right) b_{\mathrm{XX} \rightarrow X}^{\dagger}(t)|\operatorname{vac}\rangle\langle\operatorname{vac}| b_{\mathrm{XX} \rightarrow X}\left(t^{\prime}\right),
\end{aligned}
$$

where

$$
\rho\left(t, t^{\prime}\right)=\gamma_{\mathrm{XX}} e^{\mathrm{i} \omega_{\mathrm{XX} \rightarrow X}\left(t^{\prime}-t\right)} e^{-\gamma_{\mathrm{XX}}\left(t+t^{\prime}\right) / 2} e^{-\gamma_{X}\left|t-t^{\prime}\right| / 2} .
$$

Equation (2) shows that the state of the photon from the transition $|\mathrm{XX}\rangle \rightarrow|X\rangle$, while generally being a nonseparable state, becomes separable if the decay rate of the intermediate state vanishes. The nonseparability of the emitted photon immediately limits the indistinguishability of photons emitted from such a cascade, since for the emitted photons to be identical they must be describable as pure states and thus separable. The separability of this single-photon state can be quantified by its trace purity $\mathbb{P}$ which can be analytically evaluated using Eq. (2) [26]:

$$
\mathbb{P}=\operatorname{Tr}_{b_{\mathrm{XX} \rightarrow X}}\left[\rho^{2}\right]=\frac{\gamma_{\mathrm{XX}}}{\gamma_{\mathrm{XX}}+\gamma_{X}} .
$$

For QDs where typically $\gamma_{\mathrm{XX}}=2 \gamma_{X}$, the maximum achievable trace purity is limited to $\sim 0.66$. We would like to note that Eq. (3) is independent of the separation time between the emitted photons. It gives an upper bound for the indistinguishability of photons from a three-level system with perfect coherence. Additional spectral diffusion will reduce the achievable indistinguishability further.

The theoretical trace purity describes the indistinguishability of the emitted single photons, but cannot be measured directly experimentally. A more experimentally accessible quantity is the interference visibility parameter $v$ extracted from two-photon interference experiments $[27,28]$. However, for systems with negligible emission with photon numbers $>1$, it has been recently shown that $\mathbb{P}$ and $v$ are identical [27] and, consequently, $v$ is a measure for the indistinguishability of the emitted photons.

In the following we compare a quantum two-level system and a three-level quantum ladder system, by measuring the second-order coherence and two-photon interference of four different semiconductor QDs. Our two-level quantum system is an exciton state in a semiconductor QD, which is directly addressed in a pure $s$-shell resonant excitation scheme, depicted in Fig. 1(a). When the system recombines back to the ground state, a resonance fluorescence photon is emitted. The experimentally investigated three-level quantum ladder system is the biexcitonexciton cascade of semiconductor QDs. In this system, the biexciton state is resonantly addressed in a two-photon process (two-photon excitation) and recombines via the exciton state into the ground state resulting in the characteristic biexciton-exciton cascade, shown in Fig. 1(b). This cascade is crucial to generate entangled photon pairs and has been extensively studied in literature [29-31].

We use GaAs/AlGaAs QDs grown via the dropletetching method [32]. A detailed description of the sample structure can be found in Ref. [22]. Remarkably these QDs currently hold the record for highest degree of entanglement when generating polarization entangled photon pairs [33], lowest multiphoton emission probability [34], and are among the brightest entangled photon sources to date $[35,36]$.

All experiments are performed in a confocal microphotoluminescence spectroscopy setup [37] (see Supplemental Material [38] for more details and a schematic, which includes Refs. [39,40]). The second-order autocorrelation is measured in a Hanbury Brown-Twiss type experiment whereas the two-photon interference visibility is measured using a HongOu-Mandel setup.

The spectrum of the exciton under pure $s$-shell resonant excitation [Fig. 1(c)] consists of a single sharp 
(a)

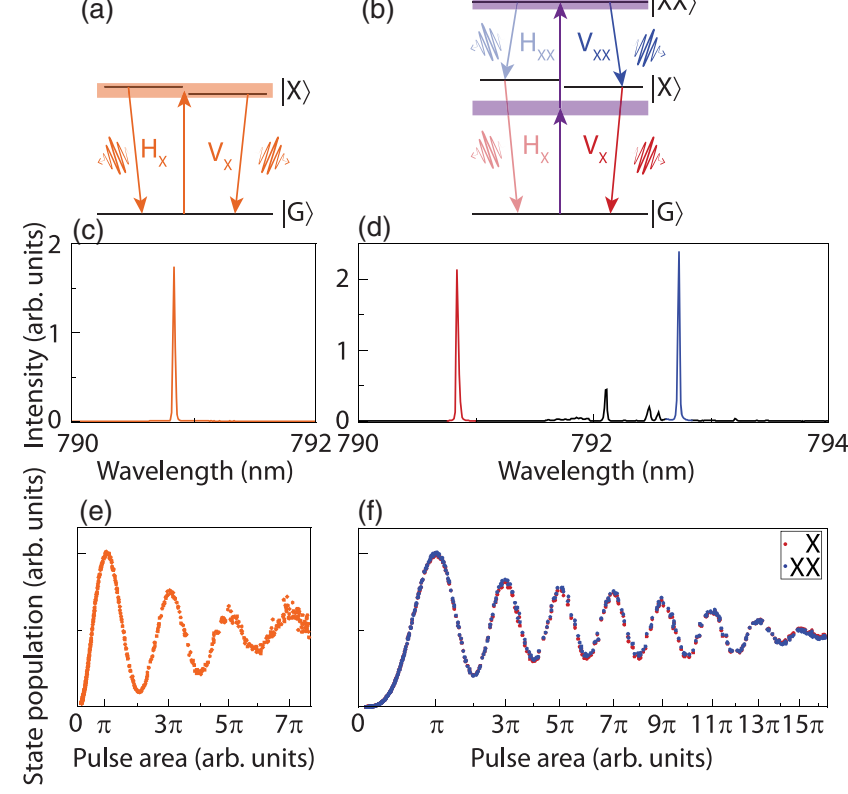

FIG. 1. Characterization of the QD under s-shell resonant excitation (a),(c),(e) and two-photon excitation (b),(d),(f). (a) $s$-shell resonant excitation scheme. The exciton level $(|X\rangle)$ is directly addressed by the excitation laser. Under recombination into the ground state $(|G\rangle)$ a resonance fluorescence (RF) photon is emitted. (b) Two-photon excitation (TPE) scheme. The biexciton state $(|\mathrm{XX}\rangle)$ is resonantly driven via a two-photon process, which recombines via the exciton state into the ground state, resulting in a biexciton (blue)-exciton (red) cascade. The $\mathrm{H}$ polarized photons are shaded to illustrate that one polarization component is suppressed by the cross-polarization setup. (c) Resonance fluorescence spectrum of the exciton. (d) Two-photon excitation spectrum with the exciton (red) at lower wavelength compared to the biexciton (blue). Excitation laser power-dependent Rabi oscillation up to a pulse area of (e) $7 \pi$ under $s$-shell resonant excitation and (f) $16 \pi$ for twophoton excitation.

peak. Power-dependent resonance fluorescence spectroscopy [Fig. 1(e)] reveals clean Rabi oscillation (see Supplemental Material [38] for analysis) up to $7 \pi$ proving the coherence of this excitation scheme. The same measurements for the exciton and biexciton under two-photon excitation are shown in Figs. 1(d) and 1(f). In the spectrum, two lines for the exciton (red) and biexciton (blue) can be seen with a wavelength difference of $1.9 \mathrm{~nm}(3.7 \mathrm{meV})$, which stems from the Coulomb interaction between the two excitons, resulting in an energetically lower (longer wavelength) biexciton photon. In between those two excitonic lines are some other lines stemming from the QD, which are additionally spectrally filtered for the following correlation measurements by the transmission spectrometer. In this excitation scheme, Rabi oscillations up to $16 \pi$ can be distinguished. The difference to the s-shell resonant excitation stems from the fact that here the laser can be filtered spectrally in addition to cross polarization, allowing much
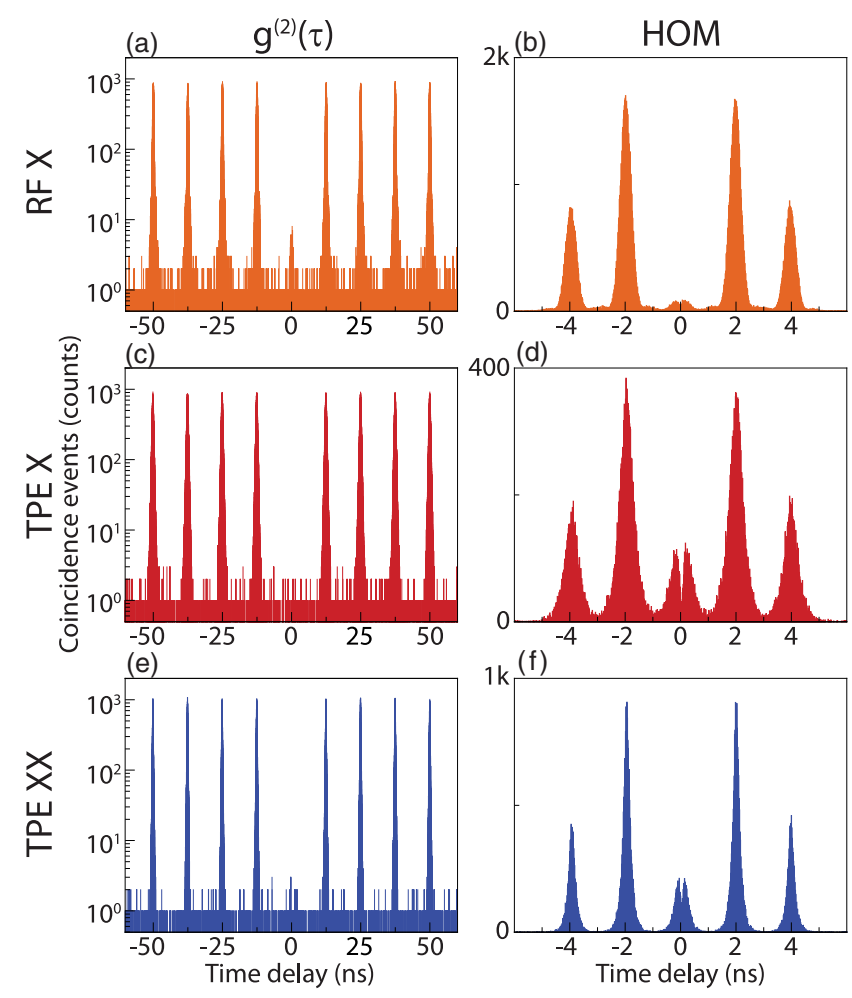

FIG. 2. Second-order intensity correlation histogram of the (a) exciton $(X)$ under $s$-shell resonant excitation (RF) with $\mathrm{g}_{\mathrm{RF}, X}^{(2)}(0)=(8.16 \pm 0.55) \times 10^{-3}$, two-photon excitation (TPE) of the (c) exciton with $\mathrm{g}_{\mathrm{TPE}, X}^{(2)}(0)=(9.13 \pm 1.61) \times 10^{-4}$ and (e) the biexciton $(\mathrm{XX})$ with $\mathrm{g}_{\mathrm{TPE}, \mathrm{XX}}^{(2)}(0)=(1.79 \pm 0.30) \times 10^{-3}$. Hong-Ou-Mandel histograms of the (b) exciton emission under resonant excitation $v_{\mathrm{RF}, X}=92.3 \pm 0.1 \%$, two-photon excitation of the (d) exciton with $v_{\mathrm{TPE}, X}=56.7 \pm 0.6 \%$ and (f) the biexciton $v_{\mathrm{TPE}, \mathrm{XX}}=60.0 \pm 0.5 \%$.

higher excitation powers. All further measurements are performed with an excitation power corresponding to a pulse area $\pi$, where the system is maximally inverted. Figure 2 shows correlation measurements for both quantum systems. The second-order autocorrelation function is shown in Figs. 2(a),2(c), and 2(e) in a semilogarithmic plot. For all measurements, the peak at time delay zero is strongly suppressed proving almost background-free single-photon emission. Exact results are shown in Table 1 of the Supplemental Material [38]. For all four QDs we investigated, the measurements under two-photon excitation show lower values than for pure s-shell resonant excitation, which we attribute to the suppressed reexcitation processes [34,41].

Next, we investigate the indistinguishability by measuring the two-photon interference of two consecutively emitted photons in a Hong-Ou-Mandel type experiment under the same excitation conditions as above. The measurement result is presented in Figs. 2(b),2(d), and 2(f), where the suppression of the center peak is a measure for the visibility $v$ and characterizes the indistinguishability of 
two photons. The experimental methods and results of all QDs under both excitation schemes are summarized in Table 1 of the Supplemental Material [38]. The raw Hong-Ou-Mandel visibility is above $90 \%$ for all QDs under pure $s$-shell resonant excitation, whereas it maximally reaches $64 \%$ under two-photon excitation, consistent with theoretical predictions for a cascaded emission as discussed above.

In order to obtain a deeper insight into how the biexcitonexciton cascade results in a reduced Hong-Ou-Mandel visibility, we performed quantum-optical simulations using the Quantum Toolbox in PYTHON (QuTiP) [42]. We model the cascade as a three-level quantum ladder system and use a quantum-optical master equation approach described in detail in Ref. [43]. These simulations go beyond the analytical theoretical considerations above as they take into account the excitation laser pulse width as well as dephasing. We neglect the fine structure splitting in the simulation since one fine structure channel is suppressed due to the cross-polarized resonance fluorescence setup. The Hamiltonian in the rotating frame at the laser frequency then reads

$$
H(t)=\frac{[\mu E(t)]^{2}}{2 E_{b}}(|G\rangle\langle\mathrm{XX}|+| \mathrm{XX}\rangle\langle G|),
$$

where $\mu$ is the electric dipole moment, $E(t)$ the electric field, and $E_{b}$ is the binding energy of the biexciton. The crystal ground state $|G\rangle$ and the biexciton level $|\mathrm{XX}\rangle$ are coupled by the electromagnetic field of a Gaussian laser pulse of length (FWHM) $\tau_{X} / 50$, where $\tau_{X}$ denotes the exciton lifetime. The pulse area

$$
A(t)=\int_{0}^{t} d t^{\prime} \frac{\left[\mu E\left(t^{\prime}\right)\right]^{2}}{\hbar E_{b}}
$$

is set to $\pi$ to achieve a maximum population inversion. The two radiative decays between the three levels $|\mathrm{XX}\rangle \rightarrow|X\rangle$ and $|X\rangle \rightarrow|G\rangle$ are coupled via two collapse operators $c_{\mathrm{XX}}=\sqrt{1 / \tau_{\mathrm{XX}}} e$ and $c_{X}=\sqrt{1 / \tau_{X}} i$, where $e=|X\rangle\langle\mathrm{XX}|$ and $i=|G\rangle\langle X|$ are the lowering operators of the excited and the intermediate state. We disregard any nonradiative decay mechanisms, as they do not contribute to the photon statistics measurements. The population evolution of this system is depicted in Fig. 3(a). During one excitation pulse the biexciton population builds up, reaching nearly unity. It then decays into the intermediate exciton state under the emission of a photon. This leads to a buildup of the exciton population, which decays into the ground state emitting another photon.

Having set up the dynamical quantum system, we turn our interest to how the properties of the emitted photons depend on the ratio of the two transition lifetimes $\tau_{\mathrm{XX}} / \tau_{X}$. To investigate the single photon character, we calculate the second-order correlation function normalized by the total photon number via the equation
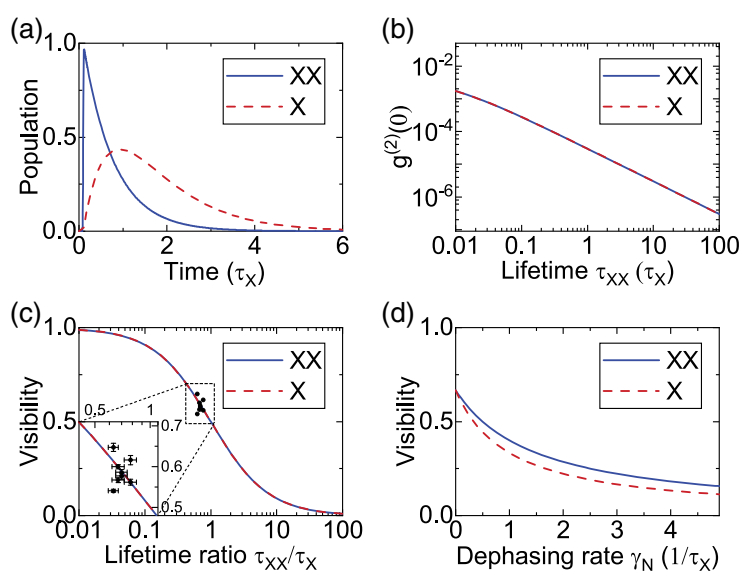

FIG. 3. Simulation of the two-photon excitation of a three-level quantum ladder system with a Gaussian pulse of duration (FHWM) $\tau_{X} / 50$ which is a commonly used pulse length in QD experiments. (a) Evolution of the biexciton (blue) and exciton (red) state population excited by a $\pi$ pulse as a function of the exciton lifetime. The exciton population builds up as the biexciton decays. (b) Simulated second-order coherence function at zero time delay $g^{(2)}(0)$ for different biexciton lifetimes. With increasing $\tau_{\mathrm{XX}}$ the $g^{(2)}(0)$ value decreases by 3 orders of magnitude for a fixed exciton lifetime $\tau_{X}$. (c) Hong-Ou-Mandel visibility of the two transitions in dependence of the lifetime ratio. The visibility decreases drastically from near unity for increasing $\tau_{\mathrm{XX}} / \tau_{X}$. Inset: Enlargement showing experimental data close to the simulated curve. (d) By adding a phenomenological pure dephasing acting with equal strength on both transitions for a fixed value of $\tau_{\mathrm{XX}} / \tau_{X}=0.5$, the obtainable Hong-Ou-Mandel visibility of both transitions decreases with a higher impact on the exciton transition visibility.

$g^{(2)}(0)=\frac{2 \int_{0}^{T} \int_{0}^{T} d t d \tau\left\langle s^{\dagger}(t) s^{\dagger}(t+\tau) s(t+\tau) s(t)\right\rangle}{\left(\int_{0}^{T} d t\left\langle s^{\dagger}(t) s(t)\right\rangle\right)^{2}}$,

where $s$ and $s^{\dagger}$ are the lowering and raising operators of the studied transition. The factor 2 is included to also take into account negative values of $\tau$. The value of $g^{(2)}(0)$ is plotted in Fig. 3(b) as a function of the lifetime $\tau_{\mathrm{XX}}$ for a constant lifetime $\tau_{X}$. With increasing $\tau_{X X}$ the value of $g^{(2)}(0)$ decreases by three orders of magnitude. As $\tau_{X}$ is fixed, increasing $\tau_{\mathrm{XX}}$ increases the total time that it takes the system to return to the ground state, which reduces reexcitation during the presence of the laser pulse and thus multiphoton emission $[34,41]$. For the dots studied in this work the lifetime ratio is $0.78 \pm 0.06>\tau_{\mathrm{XX}} / \tau_{X}>$ $0.63 \pm 0.04$ which results in values on the order of $g^{(2)}(0)=10^{-5}$, listed in Table 1 of the Supplemental Material [38] for comparison to the measured values. We continue by simulating the indistinguishability of the emitted photons in a Hong-Ou-Mandel (HOM) interferometer as outlined in Ref. [43]:

$$
g_{\mathrm{HOM}}^{(2)}(0)=\frac{1}{2} g^{(2)}(0)+\frac{1}{2}\left(1-\left|g^{(1)}(0)\right|^{2}\right) .
$$


In this normalization, for a nonideal single-photon source with $g^{(2)}(0)>0$ the value $g_{\mathrm{HOM}}^{(2)}(0)$ would increase as the beam splitter with the two detectors simultaneously represents a Hanbury Brown-Twiss experiment for each excitation pulse. As $g^{(2)}(0) \approx 0$ both in experiment and simulation for the studied parameter space, we focus on the visibility

$$
v=\left|g^{(1)}(0)\right|^{2}=\frac{2 \int_{0}^{T} \int_{0}^{T} d t d \tau\left|\left\langle s^{\dagger}(t+\tau) s(t)\right\rangle\right|^{2}}{\left(\int_{0}^{T} d t\left\langle s^{\dagger}(t) s(t)\right\rangle\right)^{2}} .
$$

The dependence of the visibility parameter with respect to the lifetime ratio is presented in Fig. 3(c). It decreases from near unity for small values of $\tau_{\mathrm{XX}} / \tau_{X}$ to almost zero for large ratios. The experimental values of approximately $60 \%$ [inset Fig. 3(c)] match the simulated curve well. Tuning the ratio over a wide range could be experimentally realized by embedding the QD into a nanophotonic resonator like photonic crystals [44], micropillars [45], bull's eye cavities [46], paraboloids [47], and even planar cavities [29], which selectively enhances or reduces the lifetimes $\tau_{\mathrm{XX}}$ and $\tau_{X}$. We would like to note that asymmetric Purcell enhancement of both transitions is preferred over Purcell suppression to maintain efficient photon extraction from the cavity. The simulation results for the lifetime ratio of our measured QDs are given in Table 1 in the Supplemental Material [38].

As discussed above, the limited visibilities for both transitions result from the entangled nature of the cascaded emission. However, it can also be explained in a simple picture by the finite lifetime of the exciton. The photons emitted by the biexciton are spectrally broadened due to the linewidth of its final state $(|X\rangle)$ resulting from its finite lifetime. On the other hand, photons emitted from the exciton state are subject to a timing jitter induced by the cascade.

Finally, we investigate how introducing additional dephasing affects the two-photon interference visibilities of both transitions. To this end, we introduce a phenomenological pure dephasing acting with equal strength on both levels, described by two additional collapse operators of the form $c_{N}^{\mathrm{XX}}=\sqrt{\gamma_{N}}|\mathrm{XX}\rangle\langle\mathrm{XX}|$ and $c_{N}^{X}=\sqrt{\gamma_{N}}|X\rangle\langle X|$. The visibility of both transitions is presented in Fig. 3(d) as a function of the dephasing rate $\gamma_{N}$ for a fixed ratio of $\tau_{\mathrm{XX}} / \tau_{X}=0.5$. With increasing dephasing rate, the visibility of both transitions decreases whereby the visibility of the exciton emission (red) is lower than that of the biexciton emission (blue). This stems from the faster biexciton emission rate compared to that of the exciton, so that the biexciton state is less affected by the same dephasing rate. This is also consistent with our experimental measurements discussed above, where typically a higher visibility was measured for the biexciton transition.

In summary, we investigated the impact of the intermediate state on the indistinguishability of cascaded photons emitted by a three-level quantum ladder system and experimentally confirmed our findings using the biexciton-exciton cascade in semiconductor QDs. The key parameter for reaching near-unity indistinguishability for photons stemming from a cascaded emission is the ratio between the lifetimes of the two excited states. Therefore, asymmetric Purcell enhancement of these states is expected to overcome the limitation on the indistinguishability of cascaded photons emitted by a three-level quantum ladder system, bringing the indistinguishability to the dephasinglimited values of a resonantly driven two-level system. This would enable the simultaneous generation of near-unity indistinguishable and entangled photon pairs required for photonic entanglement-based quantum repeater schemes (see Supplemental Material [38] for further discussion, which includes Refs. [48-51]).

This project has received funding from the European Union's Horizon 2020 research and innovation program under Grant Agreement No. 820423 (S2QUIP) and No. 899814 (Qurope), the European Research Council (ERC) under the European Union's Horizon 2020 Research and Innovation Programme (SPQRel, Grant Agreement No. 679183), the Austrian Science Fund (FWF): P 29603, P 30459, I 4320, the Linz Institute of Technology (LIT) and the LIT Lab for secure and correct systems, supported by the State of Upper Austria, the German Federal Ministry of Education and Research via the funding program Photonics Research Germany (Contract No. 13N14846), Q.Com (Project No. 16KIS0110) and Q.Link.X (Project No. 16KIS0874), the DFG via SQAM (Project No. F1 947/ 5-1), the Nanosystem Initiative Munich, the MCQST, the Knut and Alice Wallenberg Foundation grant Quantum Sensors, the Swedish Research Council (VR) through the VR grant for international recruitment of leading researchers (Ref: 2013-7152), and Linnæus Excellence Center ADOPT. K. M. acknowledges support from the Bavarian Academy of Sciences and Humanities. K. D. J. acknowledges funding from the Swedish Research Council (VR) via the starting grant HyQRep (Ref.: 2018-04812). J. V. acknowledges support from the National Science Foundation, Grant No. ECCS 1839056. K. D. J. acknowledges fruitful discussions with Stefan Schumacher. A. R. acknowledges fruitful discussions with Y. Huo, G. Weihs, R. Keil, and S. Portalupi.

*These authors contributed equally to this work.

Corresponding author.

klaus.joens@uni-paderborn.de

Present address: Department of Physics, Paderborn University, 33098 Paderborn, Germany.

[1] C. K. Hong, Z. Y. Ou, and L. Mandel, Measurement of Subpicosecond Time Intervals Between Two Photons by Interference, Phys. Rev. Lett. 59, 2044 (1987).

[2] J. I. Cirac, P. Zoller, H. J. Kimble, and H. Mabuchi, Quantum State Transfer and Entanglement Distribution 
among Distant Nodes in a Quantum Network, Phys. Rev. Lett. 78, 3221 (1997).

[3] V. Giovannetti, S. Lloyd, and L. Maccone, Quantumenhanced measurements: Beating the standard quantum limit, Science 306, 1330 (2004).

[4] H. J. Kimble, The quantum internet, Nature (London) 453, 1023 (2008).

[5] I. Aharonovich, D. Englund, and M. Toth, Solid-state single-photon emitters, Nat. Photonics 10, 631 (2016).

[6] Y.-M. He, Y. He, Y.-J. Wei, D. Wu, M. Atatüre, C. Schneider, S. Höfling, M. Kamp, C.-Y. Lu, and J.-W. Pan, 'On-demand semiconductor single-photon source with near-unity indistinguishability, Nat. Nanotechnol. 8, 213 (2013).

[7] N. Somaschi, V. Giesz, L. D. Santis, J. Loredo, M. Almeida, G. Hornecker, S. Portalupi, T. Grange, C. Antón, J. Demory, C. Gómez, I. Sagnes, N. Lanzillotti-Kimura, A. Lemaítre, A. Auffeves, A. White, L. Lanco, and P. Senellart, Nearoptimal single-photon sources in the solid state, Nat. Photonics 10, 340 (2016).

[8] X. Ding, Y. He, Z.-C. Duan, N. Gregersen, M.-C. Chen, S. Unsleber, S. Maier, C. Schneider, M. Kamp, S. Höfling, C.-Y. Lu, and J.-W. Pan, On-Demand Single Photons with High Extraction Efficiency and Near-Unity Indistinguishability from a Resonantly Driven Quantum Dot in a Micropillar, Phys. Rev. Lett. 116, 020401 (2016).

[9] J. C. Loredo, M. A. Broome, P. Hilaire, O. Gazzano, I. Sagnes, A. Lemaitre, M. P. Almeida, P. Senellart, and A. G. White, Boson Sampling with Single-Photon Fock States from a Bright Solid-State Source, Phys. Rev. Lett. 118, 130503 (2017).

[10] Y. He, X. Ding, Z.-E. Su, H.-L. Huang, J. Qin, C. Wang, S. Unsleber, C. Chen, H. Wang, Y.-M. He, X.-L. Wang, W.-J. Zhang, S.-J. Chen, C. Schneider, M. Kamp, L.-X. You, Z. Wang, S. Höfling, C.-Y. Lu, and J.-W. Pan, Time-BinEncoded Boson Sampling with a Single-Photon Device, Phys. Rev. Lett. 118, 190501 (2017).

[11] H. Wang, J. Qin, X. Ding, M.-C. Chen, S. Chen, X. You, Y.-M. He, X. Jiang, L. You, Z. Wang, C. Schneider, J. J. Renema, S. Höfling, C.-Y. Lu, and J.-W. Pan, Boson Sampling with 20 Input Photons and a 60-Mode Interferometer in a $10^{14}$-Dimensional Hilbert Space, Phys. Rev. Lett. 123, 250503 (2019).

[12] A. J. Bennett, J. P. Lee, D. J. P. Ellis, T. Meany, E. Murray, F. F. Floether, J. P. Griffths, I. Farrer, D. A. Ritchie, and A. J. Shields, Cavity-enhanced coherent light scattering from a quantum dot, Sci. Adv. 2, e1501256 (2016).

[13] M. Müller, H. Vural, C. Schneider, A. Rastelli, O. G. Schmidt, S. Höfling, and P. Michler, Quantum-Dot Single-Photon Sources for Entanglement Enhanced Interferometry, Phys. Rev. Lett. 118, 257402 (2017).

[14] M. Müller, S. Bounouar, K. D. Jöns, M. Glässl, and P. Michler, On-demand generation of indistinguishable polarization-entangled photon pairs, Nat. Photonics 8, 224 (2014).

[15] O. Benson, C. Santori, M. Pelton, and Y. Yamamoto, Regulated and Entangled Photons from a Single Quantum Dot, Phys. Rev. Lett. 84, 2513 (2000).

[16] J. Huwer, R. M. Stevenson, J. Skiba-Szymanska, M. B. Ward, A. J. Shields, M. Felle, I. Farrer, D. A. Ritchie, and R. V.
Penty, Quantum-Dot-Based Telecommunication-Wavelength Quantum Relay, Phys. Rev. Applied 8, 024007 (2017).

[17] F. Basso Basset, M. B. Rota, C. Schimpf, D. Tedeschi, K. D. Zeuner, S. F. Covre da Silva, M. Reindl, V. Zwiller, K. D. Jöns, A. Rastelli, and R. Trotta, Entanglement Swapping with Photons Generated on Demand by a Quantum Dot, Phys. Rev. Lett. 123, 160501 (2019).

[18] M. Zopf, R. Keil, Y. Chen, J. Yang, D. Chen, F. Ding, and O. G. Schmidt, Entanglement Swapping with Semiconductor-Generated Photons Violates Bell's Inequality, Phys. Rev. Lett. 123, 160502 (2019).

[19] S. Lloyd, M. S. Shahriar, J. H. Shapiro, and P. R. Hemmer, Long Distance, Unconditional Teleportation of Atomic States via Complete Bell State Measurements, Phys. Rev. Lett. 87, 167903 (2001).

[20] R. M. Stevenson, C. L. Salter, J. Nilsson, A. J. Bennett, M. B. Ward, I. Farrer, D. A. Ritchie, and A. J. Shields, Indistinguishable Entangled Photons Generated by a LightEmitting Diode, Phys. Rev. Lett. 108, 040503 (2012).

[21] M. Reindl, K. D. Jöns, D. Huber, C. Schimpf, Y. Huo, V. Zwiller, A. Rastelli, and R. Trotta, Phonon-assisted twophoton interference from remote quantum emitters, Nano Lett. 17, 4090 (2017).

[22] D. Huber, M. Reindl, Y. Huo, H. Huang, J. S. Wildmann, O. G. Schmidt, A. Rastelli, and R. Trotta, Highly indistinguishable and strongly entangled photons from symmetric GaAs quantum dots, Nat. Commun. 8, 15506 (2017).

[23] T. Huber, A. Predojević, D. Fger, G. Solomon, and G. Weihs, Optimal excitation conditions for indistinguishable photons from quantum dots, New J. Phys. 17, 123025 (2015).

[24] K. A. Fischer, R. Trivedi, V. Ramasesh, I. Siddiqi, and J. Vučković, Scattering into one-dimensional waveguides from a coherently-driven quantum-optical system, Quantum 2, 69 (2018).

[25] R. Trivedi, K. Fischer, S. Xu, S. Fan, and J. Vuckovic, Fewphoton scattering and emission from low-dimensional quantum systems, Phys. Rev. B 98, 144112 (2018).

[26] C. Simon and J.-P. Poizat, Creating Single TimeBin-Entangled Photon Pairs, Phys. Rev. Lett. 94, 030502 (2005).

[27] K. A. Fischer, R. Trivedi, and D. Lukin, Particle emission from open quantum systems, Phys. Rev. A 98, 023853 (2018).

[28] R. Trivedi, K. A. Fischer, J. Vučković, and K. Müller, Generation of non-classical light using semiconductor quantum dots, Adv. Quantum Technol., 3, 1900007 (2019).

[29] T. Huber, A. Predojević, H. Zoubi, H. Jayakumar, G. S. Solomon, and G. Weihs, Measurement and modification of biexciton-exciton time correlations, Opt. Express 21, 9890 (2013).

[30] M. Ward, M. Dean, R. Stevenson, A. Bennett, D. Ellis, K. Cooper, I. Farrer, C. Nicoll, D. Ritchie, and A. Shields, Coherent dynamics of a telecom-wavelength entangled photon source, Nat. Commun. 5, 3316 (2014).

[31] A. Orieux, M. A. M. Versteegh, K. D. Jöens, and S. Ducci, Semiconductor devices for entangled photon pair generation: A review, Rep. Prog. Phys. 80, 076001 (2017).

[32] Y. H. Huo, A. Rastelli, and O. G. Schmidt, Ultrasmall excitonic fine structure splitting in highly symmetric 
quantum dots on GaAs (001) substrate, Appl. Phys. Lett. 102, 152105 (2013).

[33] D. Huber, M. Reindl, S. F. Covre da Silva, C. Schimpf, J. Martín-Sánchez, H. Huang, G. Piredda, J. Edlinger, A. Rastelli, and R. Trotta, Strain-Tunable GaAs Quantum Dot: A Nearly Dephasing-Free Source of Entangled Photon Pairs on Demand, Phys. Rev. Lett. 121, 033902 (2018).

[34] L. Schweickert, K. D. Jöns, K. D. Zeuner, S. F. Covre da Silva, H. Huang, T. Lettner, M. Reindl, J. Zichi, R. Trotta, A. Rastelli, and V. Zwiller, On-demand generation of background-free single photons from a solid-state source, Appl. Phys. Lett. 112, 093106 (2018).

[35] J. Liu, R. Su, Y. Wei, B. Yao, S. F. C. d. Silva, Y. Yu, J. Iles-Smith, K. Srinivasan, A. Rastelli, J. Li, and X. Wang, A solid-state source of strongly entangled photon pairs with high brightness and indistinguishability, Nat. Nanotechnol. 14, 586 (2019).

[36] H. Wang, H. Hu, T.-H. Chung, J. Qin, X. Yang, J.-P. Li, R.-Z. Liu, H.-S. Zhong, Y.-M. He, X. Ding, Y.-H. Deng, Q. Dai, Y.-H. Huo, S. Höfling, C.-Y. $\mathrm{Lu}$, and J.-W. Pan, On-Demand Semiconductor Source of Entangled Photons Which Simultaneously Has High Fidelity, Efficiency, and Indistinguishability, Phys. Rev. Lett. 122, 113602 (2019).

[37] E. Schöll, L. Hanschke, L. Schweickert, K. D. Zeuner, M. Reindl, S. F. Covre da Silva, T. Lettner, R. Trotta, J. J. Finley, K. Müller, A. Rastelli, V. Zwiller, and K. D. Jöns, Resonance fluorescence of GaAs quantum dots with nearunity photon indistinguishability, Nano Lett. 19, 2404 (2019).

[38] See Supplemental Material at http://link.aps.org/ supplemental/10.1103/PhysRevLett.125.233605 for methods and summary of results for all four investigated quantum dots.

[39] A. V. Kuhlmann, J. Houel, D. Brunner, A. Ludwig, D. Reuter, A. D. Wieck, and R. J. Warburton, A dark-field microscope for background-free detection of resonance fluorescence from single semiconductor quantum dots operating in a set-and-forget mode, Rev. Sci. Instrum. 84, 073905 (2013).

[40] Z. Lin, L. Schweickert, S. Gyger, K. D. Jöns, and V. Zwiller, ETA-Extensible Timetag Analyzer (2020).

[41] L. Hanschke, K. A. Fischer, S. Appel, D. Lukin, J. Wierzbowski, S. Sun, R. Trivedi, J. Vučković, J. J. Finley, and K. Müller, Quantum dot single-photon sources with ultra-low multi-photon probability, Quantum Inf. 4, 43 (2018).

[42] J. Johansson, P. Nation, and F. Nori, QuTiP: An open-source Python framework for the dynamics of open quantum systems, Comput. Phys. Commun. 183, 1760 (2012).

[43] K. A. Fischer, K. Müller, K. G. Lagoudakis, and J. Vučković, Dynamical modeling of pulsed two-photon interference, New J. Phys. 18, 113053 (2016).

[44] P. Lodahl, A. Floris van Driel, I. S. Nikolaev, A. Irman, K. Overgaag, D. Vanmaekelbergh, and W. L. Vos, Controlling the dynamics of spontaneous emission from quantum dots by photonic crystals, Nature (London) 430, 654 (2004).

[45] J. Suffczyński, A. Dousse, K. Gauthron, A. Lemaître, I. Sagnes, L. Lanco, J. Bloch, P. Voisin, and P. Senellart, Origin of the Optical Emission within the Cavity Mode of Coupled Quantum Dot-Cavity Systems, Phys. Rev. Lett. 103, 027401 (2009).

[46] S. Kolatschek, S. Hepp, M. Sartison, M. Jetter, P. Michler, and S. L. Portalupi, Deterministic fabrication of circular Bragg gratings coupled to single quantum emitters via the combination of in-situ optical lithography and electronbeam lithography, J. Appl. Phys. 125, 045701 (2019).

[47] T. Lettner, K. D. Zeuner, E. Schöll, H. Huang, S. Scharmer, S. F. Covre da Silva, S. Gyger, L. Schweickert, A. Rastelli, K. D. Jöns, and V. Zwiller, GaAs Quantum Dot in a Parabolic Microcavity Tuned to ${ }^{87} \mathrm{Rb} \mathrm{D}_{1}$, ACS Photonics 7, 29 (2020).

[48] K. Muller, T. Kaldewey, R. Ripszam, J. S. Wildmann, A. Bechtold, M. Bichler, G. Koblmuller, G. Abstreiter, and J. J. Finley, All optical quantum control of a spin-quantum state and ultrafast transduction into an electric current, Sci. Rep. 3, 1906 (2013).

[49] Y. Kodriano, I. Schwartz, E. Poem, Y. Benny, R. Presman, T. A. Truong, P. M. Petroff, and D. Gershoni, Complete control of a matter qubit using a single picosecond laser pulse, Phys. Rev. B 85, 241304(R) (2012).

[50] T. Grange, N. Somaschi, C. Antón, L. De Santis, G. Coppola, V. Giesz, A. Lemaître, I. Sagnes, A. Auffèves, and P. Senellart, Reducing Phonon-Induced Decoherence in Solid-State Single-Photon Sources with Cavity Quantum Electrodynamics, Phys. Rev. Lett. 118, 253602 (2017).

[51] A. J. Hudson, R. M. Stevenson, A. J. Bennett, R. J. Young, C. A. Nicoll, P. Atkinson, K. Cooper, D. A. Ritchie, and A. J. Shields, Coherence of an Entangled Exciton-Photon State, Phys. Rev. Lett. 99, 266802 (2007). 